Bull. Austral. Math. Soc.

47 A 13,47 в 15

VOL. 48 (1993) [163-170]

\title{
ON JOINT SPECTRA OF NON-COMMUTING NORMAL OPERATORS
}

\section{Alan J. Pryde and Andrzej Soltysiak}

\begin{abstract}
The purpose of the paper is to show that the Harte spectrum and the bicommutant spectrum of an arbitrary $n$-tuple of normal Hilbert space operators can be obtained from the spectral set $\gamma$ introduced by McIntosh and Pryde. It is also proved that many commonly used joint spectra of an $n$-tuple of normal $m$ by $m$ matrices are equal. These results are non-commutative variants of some theorems proved by McIntosh, Pryde, and Ricker for commuting sets of operators.
\end{abstract}

Let $H$ be a complex Hilbert space. All operators considered in the sequel are assumed to be bounded linear operators on $H$. In [7, 8] McIntosh and Pryde introduced a notion of a spectral set $\gamma(T)$ associated to each $n$-tuple $T=\left(T_{1}, \ldots, T_{n}\right)$ of operators and defined as follows:

$$
\gamma(T)=\left\{\left(\lambda_{1}, \ldots, \lambda_{n}\right) \in \mathbb{R}^{n}: \sum_{j=1}^{n}\left(T_{j}-\lambda_{j}\right)^{2} \text { is not invertible in } \mathcal{B}(H)\right\}
$$

(Here we write for simplicity $T_{j}-\lambda_{j}$ instead of $T_{j}-\lambda_{j} I$.) This set has proved useful not only in the spectral theory of self-adjoint operators but also in comparing various types of joint spectra of commuting $n$-tuples of operators (see [9]). One advantage of the set $\gamma(T)$ over other joint spectra is that it can be easily computed.

In this paper we show that the spectral set $\gamma(T)$ is also useful in the multiparameter spectral theory of normal (not necessarily commuting) operators. Moreover we prove that many known joint spectra coincide on the $n$-tuples of normal $m$ by $m$ matrices.

We recall some definitions of joint spectra. Let, as before, $T=\left(T_{1}, \ldots, T_{n}\right)$ be an $n$-tuple of operators. A point $\lambda=\left(\lambda_{1}, \ldots, \lambda_{n}\right)$ of $\mathbb{C}^{n}$ is not in the left spectrum of $T$ if there exist operators $S_{1}, \ldots, S_{n} \in \mathcal{B}(H)$ such that $\sum_{j=1}^{n} S_{j}\left(T_{j}-\lambda_{j}\right)=I$. The left spectrum of $T$ will be denoted by $\sigma_{l}(T)$. The right spectrum, $\sigma_{r}(T)$, is defined analogously. The Harte spectrum of $T$ (in $\mathcal{B}(H)$ ) is the union of the left and right joint spectra; in symbols

$$
\sigma_{H}(T)=\sigma_{l}(T) \cup \sigma_{r}(T)
$$

Received 6th October, 1992

The second named author would like to thank Alan Pryde for warm hospitality extended to him during his visit to Monash University in the second half of 1992 where this paper was written.

Copyright Clearance Centre, Inc. Serial-fee code: 0004-9729/93 \$A2.00+0.00. 
It is well-known (see [6, Theorems 2.5 and 2.4]) that

$$
\sigma_{l}(T)=\left\{\lambda \in \mathbb{C}^{n}: \inf _{\|x\|=1} \sum_{j=1}^{n}\left\|\left(T_{j}-\lambda_{j}\right) x\right\|=0\right\}
$$

(the approximate point spectrum) and

$$
\sigma_{r}(T)=\left\{\lambda \in \mathbb{C}^{n}: \sum_{j=1}^{n}\left(\left(T_{j}-\lambda_{j}\right)(H)\right) \neq H\right\}
$$

(the defect spectrum).

Let $\mathcal{S}$ be a non-empty subset of $\mathcal{B}(H)$. The commutant $\mathcal{S}^{\prime}$ of $\mathcal{S}$ is the set of all operators that commute with every element of $\mathcal{S}$. The bicommutant $\mathcal{S}^{\prime \prime}$ is the commutant of $\mathcal{S}^{\prime}$. It is clear from the definition that $\mathcal{S}^{\prime \prime}$ is a closed, unital, and inverse-closed subalgebra of $\mathcal{B}(H)$ containing the set $\mathcal{S}$. The bicommutant spectrum of an $n$-tuple $T=\left(T_{1}, \ldots, T_{n}\right)$, denoted by $\sigma^{\prime \prime}(T)$, is the Harte spectrum of $T$ in its bicommutant $\left\{T_{1}, \ldots, T_{n}\right\}^{\prime \prime}$. One can also consider the Harte spectrum of $T=\left(T_{1}, \ldots, T_{n}\right)$ in the Banach algebra $\left[T_{1}, \ldots, T_{n}\right]$ generated by $T_{1}, \ldots, T_{n}$ and the identity. Let us denote this spectrum by $\widehat{\sigma}(T)$.

To define the last joint spectrum to be used in this paper we require "noncommutative polynomials" (see [6, pp. 98-99]). By $\mathcal{P}^{(n)}$ we denote the algebra of all polynomials over $\mathbb{C}$ in non-commutative indeterminates $X_{1}, \ldots, X_{n}$. In other words, $\mathcal{P}^{(n)}$ is the free associative complex unital algebra generated by the symbols $X_{1}, \ldots, X_{n}$. An $n$-tuple of operators $\left(T_{1}, \ldots, T_{n}\right) \in \mathcal{B}(H)^{n}$ induces a homomorphism $f \mapsto f\left(T_{1}, \ldots, T_{n}\right)$ from $\mathcal{P}^{(n)}$ to $\mathcal{B}(H)$ which preserves the identity and sends each $X_{j}$ to the corresponding $T_{j}(j=1, \ldots, n)$. A system $\left(f_{1}, \ldots, f_{m}\right) \in\left(\mathcal{P}^{(n)}\right)^{m}$ will be identified with a polynomial map $f: \mathcal{B}(H)^{n} \rightarrow \mathcal{B}(H)^{m}$ which sends $\left(T_{1}, \ldots, T_{n}\right)$ to $\left(f_{1}\left(T_{1}, \ldots, T_{n}\right), \ldots, f_{m}\left(T_{1}, \ldots, T_{n}\right)\right)$. The restriction of this mapping to the scalar multiples of the unit $\mathbb{C}^{n} \subset \mathcal{B}(H)^{n}$ takes its values in $\mathbb{C}^{m} \subset \mathcal{B}(H)^{m}$ and reduces to the system of "numerical" polynomials.

The Waelbroeck spectrum of an $n$-tuple $T=\left(T_{1}, \ldots, T_{n}\right)$ (sometimes called the rational, polynomial or rationally convex joint spectrum, see $[\mathbf{1}, \mathbf{9}]$, and $[\mathbf{1 0}]$ respectively) is defined to be the set:

$$
\sigma_{\mathcal{R}}(T)=\left\{\lambda \in \mathbb{C}^{n}: f(\lambda) \in \sigma(f(T)) \text { for every } f \in \mathcal{P}^{(n)}\right\} .
$$

It is known (see [10]) that for an arbitrary $n$-tuple $T=\left(T_{1}, \ldots, T_{n}\right)$ of operators the following inclusions are true:

$$
\sigma_{H}(T) \subset \sigma^{\prime \prime}(T) \subset \sigma_{\mathcal{R}}(T) \subset \widehat{\sigma}(T) .
$$


Moreover all these spectra are compact (possibly empty) subsets of $\mathbb{C}^{\text {n }}$ (see $[\mathbf{6}, 10]$, and [11]). Notice also that for a single operator $T$ the sets $\sigma_{H}(T), \sigma^{\prime \prime}(T)$, and $\sigma_{\mathcal{R}}(T)$ coincide and are equal to the usual spectrum of this element denoted by $\sigma(T)$.

For convenience of the reader we shall state the following result (see [2, Lemma 2.4]) which will be used repeatedly in the course of the paper.

DASH'S LEMma. Let $T=\left(T_{1}, \ldots, T_{n}\right)$ be an arbitrary $n$-tuple of operators. Then

(a) $\lambda \in \sigma_{l}(T)$ if and only if $0 \in \sigma\left(\sum_{j=1}^{n}\left(T_{j}-\lambda_{j}\right)^{*}\left(T_{j}-\lambda_{j}\right)\right)$,

(b) $\lambda \in \sigma_{r}(T)$ if and only if $0 \in \sigma\left(\sum_{j=1}^{n}\left(T_{j}-\lambda_{j}\right)\left(T_{j}-\lambda_{j}\right)^{*}\right)$.

This lemma immediately implies

CoROLLARY 1. If $T=\left(T_{1}, \ldots, T_{n}\right)$ is an $n$-tuple of normal operators, then

$$
\sigma_{l}(T)=\sigma_{r}(T)=\sigma_{H}(T) .
$$

For $n$-tuples of self-adjoint operators we can prove more.

Proposition 1. Let $T=\left(T_{1}, \ldots, T_{n}\right)$ be an arbitrary $n$-tuple of self-adjoint operators. Then

$$
\sigma_{l}(T)=\sigma_{r}(T)=\sigma_{H}(T)=\sigma^{\prime \prime}(T)=\sigma_{\mathcal{R}}(T)=\widehat{\sigma}(T)=\gamma(T) .
$$

Proof: From Dash's lemma we get

$$
\sigma_{l}(T)=\sigma_{r}(T)=\sigma_{H}(T)=\gamma(T) .
$$

It is also clear that

$$
\widehat{\sigma}(T) \subset \sigma\left(T_{1}\right) \times \cdots \times \sigma\left(T_{n}\right) \subset \mathbb{R}^{n} .
$$

Hence, in view of (1), it is enough to show that $\widehat{\sigma}(T) \subset \gamma(T)$.

Suppose $\lambda \notin \gamma(T)$. This implies that the operator $S=\sum_{j=1}^{n}\left(T_{j}-\lambda_{j}\right)^{2}$ is invertible in $\mathcal{B}(H)$. As $\left[T_{1}, \ldots, T_{n}\right]$ is a $C^{*}$-algebra and $S \in\left[T_{1}, \ldots, T_{n}\right]$ there exists $V$ in $\left[T_{1}, \ldots, T_{n}\right]$ such that $V S=I$. But this gives $\sum_{j=1}^{n} V\left(T_{j}-\lambda_{j}\right)\left(T_{j}-\lambda_{j}\right)=I$. So $\lambda \notin$ $\widehat{\sigma}(T)$ and the proof is complete.

REMARKs. 1. The equality $\sigma_{l}(T)=\gamma(T)$ for $T$ an $n$-tuple of self-adjoint operators was proved in [4, Proposition 2].

2. For a commuting $n$-tuple $T=\left(T_{1}, \ldots, T_{n}\right)$ of Banach space operators, equalities (2) imply that the spectra $\sigma\left(T_{j}\right)(j=1, \ldots, n)$ are real (see [9, Theorem 1]). This 
is no longer true if the operators $T_{j}$ do not commute. To see this take the following 2 by 2 matrices: $A_{1}=\left(\begin{array}{ll}1 & 0 \\ 0 & 0\end{array}\right)$ and $A_{2}=\left(\begin{array}{cc}i / 2 & 1 \\ 1 & 0\end{array}\right)$. Then $\sigma\left(A_{1}\right)=\{0,1\}$, $\sigma\left(A_{2}\right)=\{-\sqrt{15} / 4+i / 4, \sqrt{15} / 4+i / 4\}$, but $\sigma_{l}\left(A_{1}, A_{2}\right)=\sigma_{r}\left(A_{1}, A_{2}\right)=\sigma_{H}\left(A_{1}, A_{2}\right)=$ $\sigma^{\prime \prime}\left(A_{1}, A_{2}\right)=\sigma_{\mathcal{R}}\left(A_{1}, A_{2}\right)=\widehat{\sigma}\left(A_{1}, A_{2}\right)=\gamma\left(A_{1}, A_{2}\right)=\emptyset$.

Now we proceed to the case of normal operators. We start with

LEMMA . Let $T=\left(T_{1}, \ldots, T_{n}\right)$ be an arbitrary $n$-tuple of normal operators. Then

$$
\sigma_{H}\left(T, T^{*}\right)=\left\{(\lambda, \bar{\lambda}) \in \mathbb{C}^{2 n}: \lambda \in \sigma_{H}(T)\right\}
$$

where $T^{*}=\left(T_{1}^{*}, \ldots, T_{n}^{*}\right)$ and $\bar{\lambda}=\left(\overline{\lambda_{1}}, \ldots, \overline{\lambda_{n}}\right)$.

Proof: By Theorem 3.4(i) of [6] we have

$$
\sigma_{H}\left(T, T^{*}\right) \subset\left\{(\lambda, \lambda) \in \mathbb{C}^{2 n}: \lambda \in \sigma_{H}(T)\right\}
$$

The reverse inclusion follows from the identity

$$
\sum_{j=1}^{n}\left(T_{j}-\lambda_{j}\right)^{*}\left(T_{j}-\lambda_{j}\right)+\sum_{j=1}^{n}\left(T_{j}^{*}-\overline{\lambda_{j}}\right)^{*}\left(T_{j}^{*}-\overline{\lambda_{j}}\right)=2 \sum_{j=1}^{n}\left(T_{j}-\lambda_{j}\right)^{*}\left(T_{j}-\lambda_{j}\right)
$$

and Dash's lemma.

CoRollary 2. If $T=\left(T_{1}, \ldots, T_{n}\right)$ is an arbitrary $n$-tuple of normal operators, then

$$
\left\{f(\lambda, \bar{\lambda}): \lambda \in \sigma_{H}(T)\right\} \subset \sigma_{H}\left(f\left(T, T^{*}\right)\right)
$$

for every polynomial map $f \in\left(\mathcal{P}^{(2 n)}\right)^{m}$.

Proof: This is an immediate consequence of the Lemma and Theorem 3.4(ii) of [6].

Now observe that if $T=\left(T_{1}, \ldots, T_{n}\right)$ is an $n$-tuple of normal operators and $T_{j}=$ $\operatorname{Re} T_{j}+i \operatorname{Im} T_{j}, j=1, \ldots, n$, then, in view of Corollary 2 ,

$$
\lambda \in \sigma_{H}(T) \text { implies }(\operatorname{Re} \lambda, \operatorname{Im} \lambda) \in \sigma_{H}(\operatorname{Re} T, \operatorname{Im} T)
$$

where $\operatorname{Re} T=\left(\operatorname{Re} T_{1}, \ldots, \operatorname{Re} T_{n}\right), \operatorname{Im} T=\left(\operatorname{Im} T_{1}, \ldots, \operatorname{Im} T_{n}\right), \operatorname{Re} \lambda=\left(\operatorname{Re} \lambda_{1}, \ldots, \operatorname{Re} \lambda_{n}\right)$, and $\operatorname{Im} \lambda=\left(\operatorname{Im} \lambda_{1}, \ldots, \operatorname{Im} \lambda_{n}\right)$.

Let us introduce the following notation (see [9]):

$$
\Pi(T)=(\operatorname{Re} T, \operatorname{Im} T) \text { and } p\left(z_{1}, \ldots, z_{2 n}\right)=\left(z_{1}+i z_{n+1}, \ldots, z_{n}+i z_{2 n}\right)
$$


THEOREM 1. Let $T=\left(T_{1}, \ldots, T_{n}\right)$ be an arbitrary $n$-tuple of normal operators. Then

$$
\sigma_{H}(T)=\sigma^{\prime \prime}(T)=p(\gamma(\Pi(T))) .
$$

Proof: First we prove that $\sigma_{H}(T)=p(\gamma(\Pi(T)))$. By the one-way spectral mapping property of $\sigma_{H}$ (see [6, Theorem 3.2]) and Proposition 1 we get

$$
p(\gamma(\Pi(T)))=p\left(\sigma_{H}(\Pi(T))\right) \subset \sigma_{H}(p(\Pi(T)))=\sigma_{H}(T) .
$$

On the other hand, if $\lambda \in \sigma_{H}(T)$, then $(\operatorname{Re} \lambda, \operatorname{Im} \lambda) \in \sigma_{H}(\Pi(T))$, and therefore

$$
\lambda=p(\operatorname{Re} \lambda, \operatorname{Im} \lambda) \in p\left(\sigma_{H}(\Pi(T))\right)=p(\gamma(\Pi(T)))
$$

as was to be proved.

To obtain the equality $\sigma_{H}(T)=\sigma^{\prime \prime}(T)$ it is enough to show that $\sigma^{\prime \prime}(T) \subset \sigma_{H}(T)$ as the reverse inclusion is obvious (see (1)). Suppose $\lambda \notin \sigma_{H}(T)$. By Dash's lemma the operator $S=\sum_{j=1}^{n}\left(T_{j}-\lambda_{j}\right)^{*}\left(T_{j}-\lambda_{j}\right)$ has an inverse $V$ in $\mathcal{B}(H)$. Since the operators $T_{1}, \ldots, T_{n}$ are normal, it follows from Fuglede's theorem that $S \in\left\{T_{1}, \ldots, T_{n}\right\}^{\prime \prime}$. Hence $V \in\left\{T_{1}, \ldots, T_{n}\right\}^{\prime \prime}$ and from $\sum_{j=1}^{n} V\left(T_{j}-\lambda_{j}\right)^{*}\left(T_{j}-\lambda_{j}\right)=I$ we conclude that $\lambda \notin$ $\sigma^{\prime \prime}(T)$. This completes the proof.

Remarks. 1. Theorem 1 can be viewed as a generalisation of Theorem 2 and Lemma 2 of [9] to non-commuting $n$-tuples of normal operators.

2. Example 4.2, pp. 212-213 in [1], shows that even for a commuting pair of normal operators $\left(T_{1}, T_{2}\right)$ the inclusion

$$
\sigma^{\prime \prime}\left(T_{1}, T_{2}\right) \subset \sigma_{\mathcal{R}}\left(T_{1}, T_{2}\right)
$$

can be proper.

3. One can easily modify Taylor's example [12, pp.189-191] to obtain two commuting Hilbert space operators $T_{1}, T_{2}$ (one of them not being normal) with

$$
\sigma_{H}\left(T_{1}, T_{2}\right) \neq \sigma^{\prime \prime}\left(T_{1}, T_{2}\right)
$$

So, Theorem 1 is not true when the operators $T_{1}, \ldots, T_{n}$ are not normal.

4. Since Dash's lemma and Theorem 3.4 of [6] are valid for an arbitrary $C^{*}$-algebra the same is true for the results presented above.

In the finite dimensional case we can prove the following: 
TheOREM 2. Let $A=\left(A_{1}, \ldots, A_{n}\right)$ be an arbitrary $n$-tuple of normal $m$ by $m$ matrices with complex entries. Then

$$
\begin{aligned}
& \sigma_{l}(A)=\sigma_{r}(A)=\sigma_{H}(A)=\sigma^{\prime \prime}(A)=\sigma_{\mathcal{R}}(A)=\widehat{\sigma}(A) \\
& =p(\gamma(\Pi(A)))=\left\{\text { joint eigenvalues of } A_{1}, \ldots, A_{n}\right\}
\end{aligned}
$$

Proof: By Theorem 2.6 in [6]

$$
\sigma_{l}(A)=\left\{\text { joint eigenvalues of } A_{1}, \ldots, A_{n}\right\}
$$

In view of Theorem 1 , Corollary 1 , and (1) it is enough to show that $\widehat{\sigma}(A) \subset \sigma_{H}(A)$.

If $\widehat{\sigma}(A)=\emptyset$, then we are done. If, on the other hand, $\widehat{\sigma}(A) \neq \emptyset$, then by Theorem 1 of [11] (see also Proposition 2 of [3])

$$
\widehat{\sigma}(A)=\left\{\left(\varphi\left(A_{1}\right), \ldots, \varphi\left(A_{n}\right)\right): \varphi \text { a multiplicative linear functional on }\left[A_{1}, \ldots, A_{n}\right]\right\}
$$

We always assume that multiplicative linear functionals are non-zero. Notice also that $A_{j}^{*} \in\left[A_{1}, \ldots, A_{n}\right]$ for $j=1, \ldots, n$ as $A_{j}^{*}=f_{j}\left(A_{j}\right)$ for some polynomial $f_{j}$ (see [5, p.172, Exercise 3(a)]). Therefore for an arbitrary multiplicative linear functional $\varphi$ on the algebra $\left[A_{1}, \ldots, A_{n}\right]$ we obtain

$$
\sum_{j=1}^{n}\left(A_{j}-\varphi\left(A_{j}\right)\right)^{*}\left(A_{j}-\varphi\left(A_{j}\right)\right) \subset \text { kernel } \varphi \neq\left[A_{1}, \ldots, A_{n}\right]
$$

Thus the element $\sum_{j=1}^{n}\left(A_{j}-\varphi\left(A_{j}\right)\right)^{*}\left(A_{j}-\varphi\left(A_{j}\right)\right)$ is not invertible in $\left[A_{1}, \ldots, A_{n}\right]$ and consequently not invertible in the algebra of all $m$ by $m$ matrices. An application of Dash's lemma concludes the proof.

Remarks. 1. In [4, Proposition 5], it is shown that for self-adjoint matrices $A=$ $\left(A_{1}, \ldots, A_{n}\right), \gamma(A)=\left\{\right.$ joint eigenvalues of $\left.A_{1}, \ldots, A_{n}\right\}$.

2. To show that Theorem 2 is not true when the matrices are not normal take the following two 5 by 5 matrices:

$$
A_{1}=\left(\begin{array}{lllll}
0 & 1 & 0 & 0 & 0 \\
0 & 0 & 0 & 0 & 0 \\
0 & 0 & 0 & 1 & 0 \\
0 & 0 & 0 & 0 & 1 \\
0 & 0 & 0 & 0 & 0
\end{array}\right) \text { and } A_{2}=\left(\begin{array}{lllll}
0 & 0 & 1 & 0 & 0 \\
1 & 0 & 0 & 0 & 0 \\
0 & 0 & 0 & 0 & 0 \\
0 & 0 & 0 & 0 & 0 \\
0 & 0 & 0 & 1 & 0
\end{array}\right)
$$


Then (see [10, Example 1])

$$
p\left(\gamma\left(\Pi\left(A_{1}, A_{2}\right)\right)\right)=\sigma_{l}\left(A_{1}, A_{2}\right)=\sigma_{r}\left(A_{1}, A_{2}\right)=\sigma_{H}\left(A_{1}, A_{2}\right)=\sigma^{\prime \prime}\left(A_{1}, A_{2}\right)=\emptyset
$$

while

$$
\sigma_{\mathcal{K}}\left(A_{1}, A_{2}\right)=\widehat{\sigma}\left(A_{1}, A_{2}\right)=\{(0,0)\}
$$

3. To give another example showing that it can happen that $\sigma_{H}\left(A_{1}, A_{2}\right) \neq$ $\sigma^{\prime \prime}\left(A_{1}, A_{2}\right)$, consider the following two 3 by 3 matrices:

$$
A_{1}=\left(\begin{array}{ccc}
1 & -1 & 0 \\
0 & 1 & 0 \\
0 & 0 & 0
\end{array}\right) \text { and } A_{2}=\left(\begin{array}{ccc}
0 & 0 & 0 \\
0 & 1 & -1 \\
0 & 0 & 2
\end{array}\right)
$$

It is a matter of simple computation to show that

$$
\begin{aligned}
p\left(\gamma\left(\Pi\left(A_{1}, A_{2}\right)\right)\right) & =\emptyset, \sigma_{l}\left(A_{1}, A_{2}\right)=\{(1,0)\}, \sigma_{r}\left(A_{1}, A_{2}\right)=\{(0,2)\} \\
\sigma_{H}\left(A_{1}, A_{2}\right) & =\{(1,0),(0,2)\} \neq \sigma^{\prime \prime}\left(A_{1}, A_{2}\right)=\sigma_{\mathcal{R}}\left(A_{1}, A_{2}\right) \\
& =\widehat{\sigma}\left(A_{1}, A_{2}\right)=\{(1,0),(1,1),(0,2)\} .
\end{aligned}
$$

Finally we prove

Proposition 2. For an arbitrary $n$-tuple $A=\left(A_{1}, \ldots, A_{n}\right)$ of $m$ by $m$ complex matrices, $\sigma_{\mathcal{R}}(A)=\hat{\sigma}(A)$.

Proof: From the proof of Theorem 2 it is clear that it is enough to show

$\left\{\left(\varphi\left(A_{1}\right), \ldots, \varphi\left(A_{n}\right)\right): \varphi\right.$ a multiplicative linear functional on $\left.\left[A_{1}, \ldots, A_{n}\right]\right\} \subset \sigma_{\mathcal{R}}(A)$.

Let $\varphi:\left[A_{1}, \ldots, A_{n}\right] \rightarrow \mathbb{C}$ be an arbitrary multiplicative linear functional and $f$ an arbitrary polynomial in $\mathcal{P}^{(n)}$. Then we get

$$
f\left(\varphi\left(A_{1}\right), \ldots, \varphi\left(A_{n}\right)\right)=\varphi\left(f\left(A_{1}, \ldots, A_{n}\right)\right) \in \hat{\sigma}\left(f\left(A_{1}, \ldots, A_{n}\right)\right)=\sigma\left(f\left(A_{1}, \ldots, A_{n}\right)\right)
$$

and the result follows.

\section{References}

[1] R.E. Curto, 'Connections between Harte and Taylor spectra', Rev. Roumaine Math. Pures Appl. 31 (1968), 203-215.

[2] A.T. Dash, 'Joint essential spectra', Pacific J. Math 64 (1976), 119-128.

[3] C.-K. Fong and A. Soltysiak, 'Existence of a multiplicative functional and joint spectra', Studia Math. 81 (1985), 213-220. 
[4] G. Greiner and W.J. Ricker, 'Joint spectral sets and commutativity of systems of $2 \times 2$ selfadjoint matrices'. Preprint .

[5] P.R. Halmos, Finite-dimensional vector spaces (D. Van Nostrand, Princeton, 1958).

[6] R.E. Harte, 'Spectral mapping theorems', Proc. Roy. Irish Acad. Sect. A 72 (1972), 89-107.

[7] A.G.R. Mclntosh and A.J. Pryde, 'The solutions of systems of operator equations using Clifford algebras', Proc. Centre Math. Anal. Austral. Nat. Univ. 9 (1985), 212-220.

[8] A.G.R. McIntosh and A.J. Pryde, 'A functional calculus for several commuting operators', Indiana Univ. Math. J. 36 (1987), 421-439.

[9] A.G.R. McIntosh, A.J. Pryde and W.J. Ricker, 'Comparison of joint spectra for certain classes of commuting operators', Studia Math. 88 (1988), 23-36.

[10] V. Müller and A. Sołtysiak, 'On the largest generalized joint spectrum', Comment. Math. Univ. Carolin. 29 (1988), 255-259.

[11] V. Müller and A. Sottysiak, 'Spectrum of generators of a noncommutative Banach algebra', Studia Math 93 (1989), 87-95.

[12] J.L. Taylor, 'A joint spectrum for several commuting operators', J. Funct. Anal. 6 (1970), 172-191.

Department of Mathematics

Monash University

Clayton Vic 3168

Australia
Institute of Mathematics

A. Mickiewicz University Matejki 48/49, 60769 Poznań

Poland 